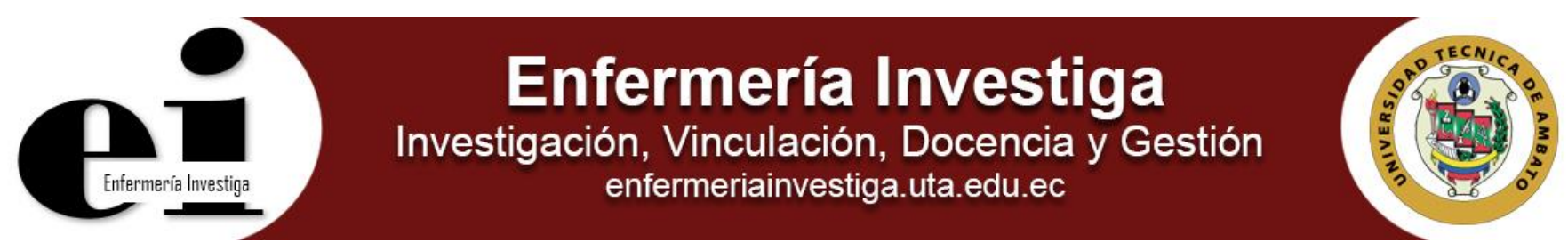

Artículo revisión

\title{
PROBLEMAS MAS FRECUENTES EN PACIENTES ONCOLOGICOS
}

\author{
Meri Ordoñez Sigcho ${ }^{1}$ \\ ${ }^{1}$ Universidad Técnica Particular de Loja - Loja - Ecuador
}

Ordoñez M. Problemas más frecuentes en pacientes oncológicos. Enferm Inv. 2019; 4(2):8-13

2477-9172 / 2550-6692 Derechos Reservados @ 2019 Universidad Técnica de Ambato, Carrera de Enfermería. Este es un artículo de acceso abierto distribuido bajo los términos de la Licencia Creative Commons, que permite uso ilimitado, distribución y reproducción en cualquier medio, siempre que la obra original es debidamente citada.

\section{Historia:}

Recibido: 15 octubre 2018

Revisado: 14 noviembre 2018

Aceptado: 18 marzo 2019

Palabras Claves: Pacientes oncológicos, calidad de vida, bien morir, control de síntomas, dolor, analgésicos.

Keywords: Oncological patients, quality of life, good to die, symptom control, pain, analgesics

\section{Resumen}

Dada el rol esencial de la enfermera en ayudar en la recuperación de salud o asistir a un bien morir, en ambos casos con altos niveles de calidad. El presente artículo expone, con el objetivo mencionado, como abordar problemas y cuidados en pacientes oncológicos. Inicia con los principios generales del control de síntomas, el enfrenamiento al dolor y su escala de analgésicos de la OMS, así como coadyuvantes del dolor y finalmente terapias antiálgicas. Finalmente se detalla el tratamiento farmacológico y no farmacológico de ciertas enfermedades comunes a pacientes oncológicos

\begin{abstract}
Given the essential role of the nurse in helping in the recovery of health or assisting a good to die, in both cases with high levels of quality. The present article exposes, with the mentioned objective, how to approach problems and care in oncological patients. It starts with the general principles of symptom control, pain control and its scale of WHO analgesics, as well as pain aids and finally antiallergic therapies. Finally, the pharmacological and non-pharmacological treatment of certain diseases common to cancer patients is detailed.
\end{abstract}




\section{Introducción}

Según Virginia Henderson "el rol esencial de la enfermera consiste en ayudar al individuo sano o enfermo en el mantenimiento o recuperación de la salud, o bien asistirlo en sus últimos momentos para que tenga una muerte feliz" (Cruz Hernández 2004)

Por tanto el objetivo de los cuidados de enfermería a la persona con enfermedad oncológica es contribuir a mantener el nivel más alto de calidad de vida y ayudarle a resolver o minimizar sus necesidades, así como brindar apoyo para que viva tan confortablemente como sea posible y contribuir a que muera con dignidad.

Los pacientes con cáncer presentan multitud de problemas, el grupo de síntomas más frecuente lo constituye el llamado síndrome general que incluye el dolor que afecta al $96 \%$ de los enfermos, mientras que la astenia, anorexia y pérdida de peso está presente en $90 \%$ de los pacientes. (Cruz Hernández 2004)

Por lo que es pertinente abordar estos problemas y contribuir con cuidados de enfermería, a minimizar sus dolencias, a aliviar sus temores y sobre todo proporcionar acompañamiento que mitigue sus temores.

En este capítulo se describen algunos principios y cuidados para poder proporcionar y explicar a los pacientes y familiares sobre los problemas más frecuentes en pacientes oncológicos.

\section{Principios generales del control de síntomas:}

- Previo a cualquier tratamiento se debe conocer la etiología del síntoma, si es secundario a los tratamientos o debido a la propia enfermedad.

- Valorar la necesidad de tratar un determinado síntoma dependiendo de la fase evolutiva de la enfermedad y de la situación del paciente.

- Explicar las causas de los síntomas al paciente y familia con términos sencillos.

- Elegir tratamientos fáciles de cumplir y siempre que sea posible elegir la vía oral.

- Iniciar los tratamientos lo antes posible y si se puede adelantarnos a los síntomas y tratar de evitar que se hagan crónicos.

- No limitarse al uso de fármacos, incluir medidas no farmacológicas.

- Evitar en lo posible la polimedicación, restringir si se puede a 405 fármacos e ir retirando los innecesarios, para lo que hay que revisar continuamente el tratamiento.

\section{ENFRENTAMIENTO DEL DOLOR}

"Es una sensación molesta y aflictiva, más o menos intensa, de una parte, del cuerpo por causa interior o exterior, que se experimenta de forma prolongada".

Según su etiología el dolor se puede clasificar en: nociceptivo (somático y visceral) y neuropático (central y periférico), también existen dolores "psicógenos y morales" por lo que es útil el concepto de "dolor total" de C. Saunders (2003), que engloba el estímulo físico nocivo y los factores psicológicos, sociales y espirituales.

El dolor en los pacientes con cáncer representa algo más que una sensación nociceptiva e incluye aspectos que tienen que ver con la condición humana, como la personalidad, la afectividad y las emociones, y las relaciones psicosociales, por lo que es importante un modelo de intervención multifactorial que comprenda medidas farmacológicas, psicoterapéuticas y rehabilitadoras, entre otras. Los tratamientos psicosociales orientados a resolver variables de la esfera psicológica mejoran el dolor mientras que los tratamientos dirigidos a la nocicepción tienen efectos psicológicos beneficiosos. (Saunders 2003)

La comunicación con el paciente y su familia es determinante para el éxito del tratamiento, el informar sobre el dolor y la forma de tratarlo, involucrando al paciente en su manejo, se acompaña de una mejor respuesta al tratamiento.

\section{Escala analgésica de la OMS}

Los analgésicos utilizados en la práctica clínica han sido clasificadas por la Organización Mundial de la Salud como:

Nivel 1: No opioides con/sin adyuvantes.

Nivel 2: Opioides débiles con/sin adyuvantes.

Nivel 3: Opioides potentes con/sin adyuvantes.

Nivel 1: Paracetamol, ácido acetilsalicílico, antiinflamatorios no esteroideos, metamizol. Los antiinflamatorios no esteroideos tienen techo analgésico, por encima de éste no producen más analgesia, pero sí más efectos secundarios. Junto con el ácido acetilsalicílico son especialmente útiles en el dolor óseo. El metamizol es más eficaz en dolores viscerales por su actividad espasmolítico. 
La dosis es prescrita por el médico, sin embargo, es necesario conocer las dosis que se pueden administrar, la dosis máxima de ácido acetilsalicílico y Paracetamol 1 gr. cada 4 horas. Metamizol: 8 gr. diarios. lbuprofeno: 2.400 mgr. diarios. Naproxeno: $1.250 \mathrm{mgr}$. diario.

Nivel 2: Codeína, Dihidrocodeina, Tramal. Los efectos secundarios son:

estreñimiento, náuseas, vómitos, mareos. El tramal tiene una potencia analgésica ligeramente superior a la codeína. Dosis máxima: Codeína: 60 mg. cada 4 horas. Dihidrocodeina 120mg cada 12

horas. Tramal $400 \mathrm{mg}$ al día.

Nivel 3: Morfina, Metadona, Fentanilo.

La morfina es el fármaco de elección en el dolor severo. No tiene techo

analgésico. Los efectos secundarios son: estreñimiento, náuseas, vómitos, sudoración, confusión, boca seca; conviene adelantarse a ellos. Se puede administrar por cualquier vía. Al iniciar el tratamiento se debe utilizar la morfina de liberación inmediata (cada 4 horas) para ajustar dosis, cuando se conozca la dosis total se pasará a la morfina de liberación controlada; el síndrome de abstinencia a la retirada de morfina se corrige haciéndolo de forma gradual y controlada; es muy raro que se produzca adicción en estos pacientes. La morfina retardada no se puede partir ni machacar.

\section{Fármacos adyuvantes en el tratamiento del dolor:}

Son fármacos que no tienen un efecto analgésico propio, pero que modifican los

factores que pueden aumentar el umbral del dolor, se suelen administrar junto a los analgésicos clásicos. Los principales son:

Antidepresivos, anticonvulsivantes, neurolépticos, corticoides que además de aliviar el dolor, elevan el estado de ánimo, aumentan el apetito, la fuerza y la sensación de bienestar y tienen actividad antiinflamatoria y antiemética.

\section{Medidas no farmacológicas:}

En muchas ocasiones el dolor está producido por problemas no relacionados

exclusivamente con el tumor, como es la debilidad, la inmovilidad, la tensión muscular, el deterioro físico. Recordemos el concepto anteriormente mencionado de "dolor total", muchos de estos dolores responden bien a medidas físicas sencillas añadidas a los analgésicos. Estas medidas serán proporcionadas por personal de salud, el propio enfermo o la familia tras entrenamiento.

Calor: Relaja los músculos y proporciona sensación de confort, puede utilizarse

a través de baños templados, botellas de agua caliente, ropas templadas, manta eléctrica a temperatura mínima, se debe tener cuidado de que no se produzcan quemaduras y evitarlo en zonas de radioterapia aunque se haya acabado el tratamiento.

Frío: Es más eficaz que el calor, produce un alivio más rápido y prolongado

aunque los pacientes prefieran éste último. Está indicado en dolor que procede de inflamación de tejidos. Puede usarse en forma de compresas frías o con hielo, bien de forma breve o con masaje.

Mentol: Altera la sensibilidad de la piel en la zona donde se aplica y produce la

sensación inicial de calor y posteriormente frío por evaporación, su efecto aumenta con mayores concentraciones pero puede resultar incómodo por su fuerte olor. Usado durante la noche ayuda a facilitar el sueño.

Masaje: El contacto físico, es en sí mismo es positivo para el paciente, el masaje

proporciona un efecto placentero, los efectos secundarios son mínimos, solo está contraindicado, si se realiza de forma vigorosa, cuando existen metástasis en huesos largos por el riesgo de fractura patológica. El masaje suave en la espalda o en los pies o manos resultan muy agradables a los pacientes.

Vibración: Es una forma más vigorosa de masaje con pocos riesgos de

producir lesiones, tiene el inconveniente del costo y a veces del ruido que producen los aparatos vibradores 0 masajeadores.

Relajación: Dado que existe relación entre dolor, tensión muscular y ansiedad que contribuyen a empeorar el dolor. La relajación rompe este círculo vicioso, es más efectiva si se utiliza de forma preventiva o cuando el dolor no es muy intenso. Existen varias técnicas de relajación, pero todas tienen en común el objetivo de aprender a relajar los músculos, a dejarlos sin tensión, para luego entrenar la mente para dejar de tener pensamientos reiterativos. 
Movilizaciones activas o pasivas: En pacientes muy débiles es frecuente la aparición de dolor por la inmovilidad. Practicar movimientos activos o pasivos de todas las articulaciones de manera suave entre 5 y 10 veces, una o dos veces al día, puede ayudar a controlar este tipo de dolores o prevenir su aparición, además de conservar la movilidad articular.

Las medidas descritas son eficaces para ayudar a controlar el dolor, pero deben valorarse y aplicarse de forma individualizada. También deben tenerse en cuenta medidas de confort general, como buscar posiciones confortables ayudándose de almohadas o cojines blandos, evitar movimientos bruscos, distraer al paciente involucrándole en actividades agradables (hace que deje de pensar en el dolor), estas actividades se deberán planificar cuando el paciente se sienta mejor y esté más despierto. Son útiles también las técnicas de respiración que se basan en respiraciones profundas y el utilizar imágenes relajantes.

En algunos casos a pesar de todo lo comentado para el control del dolor hay que recurrir a terapias antiálgicas más agresivas como bloqueos nerviosos, o procedimientos neuroquirúrgicos.

\section{NÁUSEAS}

Se definen las náuseas como sensación desagradable, en forma de oleada, en la parte posterior de la garganta, el epigastrio o por todo el abdomen que puede o no terminar en vómito.

Las náuseas y vómitos están presentes hasta en $60 \%$ de los pacientes con cáncer avanzado y pueden deberse a múltiples causas: secundarios al cáncer, secundarios al tratamiento, otros motivos como úlcera péptica, olores, presión para que coma, etc.

Es muy importante hacer un buen diagnóstico para lo que habrá que investigar sus características: cuando ocurren, cantidad, contenido, los síntomas asociados; es importante realizar una correcta exploración en busca de ruidos abdominales, distensión abdominal, vientre en tabla, hepatomegalia. Revisar los hábitos dietéticos, el ritmo intestinal y la higiene oral.

Hay algunas preguntas que pueden sugerirnos un diagnóstico, si existe dolor:

gastritis erosiva, si hay mucha sed: hipercalcemia, si hay hipo: uremia, si lleva 4-5 días sin deposición: náuseas debidas a estreñimiento; vómitos sin náusea: aumento de la presión intracraneal.

\section{Medidas no farmacológicas:}

- Enseñar a disminuir el reflejo del vómito: respirar profundamente y tragar, inspirar por la nariz y espirar lentamente por la boca.

- Explicar las causas si se conocen.

- Indicar que los alimentos deben ofrecerlos en pequeñas cantidades en varias tomas repetidas.

- Procurar un entorno agradable y evitar los olores fuertes y la visión de objetos desagradables.

- Posición sentado o semisentado, sin nada que presione el abdomen, realizando los cambios de postura de forma lenta.

\section{Tratamiento farmacológico:}

- Vómitos producidos por opioides: Haloperidol; Metoclopramida.

- Por quimioterapia: Ondansetron; Dexametasona; Lorazepan.

- Por aumento de la presión intracraneal: Dexametasona.

- Estreñimiento: Laxantes.

- Hipercalcemia: Hidratar y bifosfonatos.

- Compresión gástrica: Corticoides; Metoclopramida.

\section{ESTREÑIMIENTO}

Se define como la emisión infrecuente de heces, dos o menos veces a la semana, de consistencia dura y seca que el paciente tiene dificultad para expulsar.

Puede aparecer en $80-90 \%$ de los pacientes con cáncer. Las principales causas son: Inactividad, debilidad, disminución de la ingesta de líquidos y sólidos y de fibra en la dieta, alteraciones metabólicas (hipercalcemia, hipopotasemia), obstrucción mecánica por tumor, lesiones locales a nivel anal, dificultad para acceder al baño y falta de privacidad, secundaria a tratamiento con (opioides, anticolinérgicos, fenotiacina).

Las complicaciones del estreñimiento pueden ser: dolor, obstrucción intestinal, diarrea por rebosamiento, disfunciones urinarias.

Se debe valorar: frecuencia y consistencia, síntomas asociados como náuseas, vómitos, dolor, distensión abdominal, retención urinaria, inquietud, confusión, ventoseo, ruidos intestinales. 
En el estreñimiento de varios días de evolución se debe realizar tacto rectal para valorar el estado de la ampolla rectal (llena, vacía, consistencia de las heces).

\section{Medidas no farmacológicas:}

Aumentar la ingesta de líquidos, en forma de agua y frutas y si es posible, ya que no suelen tolerarlo dado que aumentan la sensación de saciedad se puede tratar de aumentar los alimentos con fibra, vigilando que no exista obstrucción intestinal.

Responder inmediatamente a los deseos de defecar.

Permitir la intimidad para ir al baño.

Procurar establecer horario regular.

Aumentar la actividad física dentro de las posibilidades.

Revisar y cambiar si se puede los fármacos que provocan estreñimiento.

Prevenir la retención de gases evitando el consumo de bebidas gaseosas y alimentos flatulentos, no dar a beber líquidos con sorbete.

Favorecer la expulsión de gases con movilizaciones frecuentes.

Explicar la técnica de colocación de enemas.

Cuidar la zona perianal: aplicando lubricantes en el ano, compresas frías para

aliviar el escozor, limpiar la piel con papel suave o toallitas jabonosas o con esponjas y jabones suaves.

Si se van a utilizar opioides, se deben indicar laxantes de forma profiláctica.

\section{Tratamiento farmacológico:}

Los laxantes pueden ser de varios tipos, su administración depende del problema de cada paciente, sin embargo se puede utilizar aquellos que aumentan el volumen de la masa fecal, como salvado de trigo, metilcelulosa, aunque se debe tener cuidado ya que pueden dar la sensación de llenura lo que favorece la anorexia.

Los reblandecedores de heces como lactulosa, son de ayuda, sinembargo dar dosis altas pueden producir flatulencias.

En estreñimientos pertinaces conviene utilizar laxantes de distinto tipo de acción, por ejemplo, Parafina + Senósidos +Osmóticos.

Se puede administrar vía rectal supositorios de glicerina ya que ablandan las heces duras del recto, lubricantes como el aceite de oliva en forma de enemas para desimpactar las heces.

Si existe fecaloma se aplicará un enema de limpieza con $100 \mathrm{cc}$. de aceite de oliva por la noche dos días seguidos o un enema fosfatado cada mañana o dos enemas de limpieza en días consecutivos de litro y medio de agua con aceite.

$\mathrm{Si}$, a pesar de todo ello, no es eficaz, se debe realizar una extracción manual, para ello se le indicará al paciente la maniobra que se va a realizar. Aconsejarle que respire tranquilo y de forma profunda, en posición de decúbito lateral. Lubricar abundantemente el dedo índice enguantado, introducir el dedo en el recto lo más profundo que sea posible, moviéndolo en círculo para investigar la existencia de pequeñas masas. Si el fecaloma es demasiado grande, intentaremos fragmentarlo y lo iremos extrayendo con movimientos de retracción del dedo. Repetir la maniobra hasta extraer la mayor cantidad posible de heces. No forzar nunca la maniobra, la aparición de sangrado o dolor intenso desaconseja continuar. Al finalizar se pondrá un enema para limpiar bien la ampolla rectal y se ajustará la dosis de laxante oral.

\section{CAQUEXIA:}

Este síndrome se caracteriza por pérdida de peso (grasa, músculo y proteína visceral), debilidad, náuseas y anorexia.

En las fases terminales está presente hasta en $80 \%$ de los pacientes, produce un importante sufrimiento en los pacientes y sus familias por la alteración de la imagen corporal. Así mismo, la pérdida de apetito es vivida con angustia por la familia, es importante valorar posibles causas que hacen reversible la anorexia como estreñimiento, náuseas, malestar en la boca, dolor no controlado, desequilibrio hidroelectrolítico, factores emocionales como temores o ansiedad(De Vita, 2005).

\section{Tratamiento farmacológico:}

Metoclopramida antes de las comidas y al acostarse. Alivia las náuseas y la saciedad precoz. 
Corticoides: Aumentan el apetito y la sensación de bienestar. Dexametasona

(mejora el apetito y la debilidad, no mejora el peso o el estado general);

Metilprednisolona, aumenta el apetito de forma significativa pero el beneficio desaparece a las 4-5 semanas; Prednisolona. El efecto en todos ellos es transitorio por lo que estarían indicados en pacientes con corta expectativa de vida (+- un mes)

Acetato de Megestrol: Aumenta el apetito y el peso. Está indicado en pacientes con expectativa de vida mayor a un mes. La desventaja es su elevado costo.

Cannabinoides: Dronabinol. Produce mejoría de ánimo y del apetito, siendo más útil cuando hay náuseas y en pacientes jóvenes. Efectos adversos: somnolencia, confusión, alteraciones perceptivas.

En pacientes incapaces de tragar (cáncer de cabeza, cuello, esófago) y con conservación del apetito y del estado general, estaría indicada una sonda nasogástrica o gastrostomía ya que mejora el estado nutricional pero también el grado de confort y la imagen corporal del paciente.

\section{Conflicto de intereses}

Ninguno declarado por los autores.

\section{Financiación}

Autofinanciado.

\section{Agradecimientos}

Ninguno declarado por los autores.

\section{Referencias}

1. Cruz Hernández, J.J. Lecciones de Oncología clínica. Ed. Nova-Sidonia; 2004

2. De Vita, V.T. Hellman S. Rosenberg. Cáncer: Principles and practice of oncology. 7th Edition. Lippincott Williams\&Wilkins; 2005

3. Saunders C. The evolution of palliative care. Pharos Alpha Omega Alpha Honor Med Soc; 2003. 